\title{
Abstract: Retrieval of Attenuation Values by the Augmented Likelihood Image Reconstruction in the Presence of Metal Artefacts
}

\author{
Maik Stille ${ }^{1}$, Christian Ziemann ${ }^{2}$, Florian Cremers ${ }^{2}$, Dirk Rades ${ }^{2}$, \\ Thorsten M. Buzug ${ }^{1}$ \\ ${ }^{1}$ Institute of Medical Engineering, University of Luebeck \\ ${ }^{2}$ Department of Radiotherapy, University Hospital Schleswig-Holstein, Campus \\ Luebeck \\ stille@imt.uni-luebeck.de
}

Metal implants are able to cause severe artefacts in CT images due to physical effects such as scattering, total absorption, noise, or beamharding. Typically, the reconstructed images feature dark shadows around high-density objects as well as bright and dark streaks that may reduce the diagnostic value drastically. Within an extensive evaluation, the novel algorithm Augmented Likelihood Image Reconstruction has proven to reduce occurring artefacts accurately [1]. Moreover, the quality of artefact reduction should not only be based on the ability to reconstruct the shape of anatomical structures but on the resulting attenuation values as well. A commercially available phantom, Gammex Electron Density CT Phantom Model 465, Radiation Measurements Inc, is used to investigate the ability to retrieve attenuation values of various tissue classes [2]. The phantom is equipped with two steel inserts that cause strong artefacts similar to metal implants. On a position between the two metal rods where the manifestation of artefacts is strongest, 12 different inserts with tissue equivalent attenuation values were placed. The investigated attenuation values cover a range from lung tissue with $-700 \mathrm{HU}$ to cortical bone with $1336 \mathrm{HU}$. For each insert, mean value and standard deviation are compared to reference values, which are gained from a scan with removed metal rods. The evaluation shows that the HU-values of the different tissue classes could be retrieved with errors below the standard deviation of the reference images. The lowest error could be achieved for cortical bone with a difference in the mean value of only $5 \mathrm{HU}$ and a standard deviation of $67 \mathrm{HU}$. Notably, the evaluation shows an overall drop in the standard deviation compared to the reference values. While the shape of the inserts was reconstructed accurately, this indicates an ability to reduce noise in the images.

\section{References}

1. Stille M, Kleine M, Buzug TM, et al. Augmented Likelihood Image Reconstruction. IEEE Transactions on Medical Imaging. 2016;35(1):158-173.

2. Ziemann C, Stille M, Cremers F, et al. The effects of metal artifact reduction on the retrieval of attenuation values. Journal of Applied Clinical Medical Physics. 2017;18(1):243-250. 